\title{
Synchronous chaos in the coupled system of two logistic maps
}

\author{
Guo-yi He ${ }^{\text {a }}$, Guo-wei He ${ }^{\text {b** }}$ \\ a Nanchang Institute for Aeronautical Technology, Nanchang 330034, China \\ ${ }^{\mathrm{b}}$ Laboratory for Nonlinear Mechanics, Institute of Mechanics, Chinese Academy of Sciences, Beijing 100080, China
}

Accepted 22 April 2004

\begin{abstract}
Synchronous chaos is investigated in the coupled system of two Logistic maps. Although the diffusive coupling admits all synchronized motions, the stabilities of their configurations are dependent on the transverse Lyapunov exponents while independent of the longitudinal Lyapunov exponents. It is shown that synchronous chaos is structurally stable with respect to the system parameters. The mean motion is the pseudo-orbit of an individual local map so that its dynamics can be described by the local map.
\end{abstract}

(c) 2004 Elsevier Ltd. All rights reserved.

\section{Introduction}

The object of this research is to investigate synchronous chaos in the coupled system of two Logistic maps: how temporal chaos on each map is spatially synchronized by a diffusive coupling. Since the diffusive coupling admits all spatial uniform configurations, the key problem is the stability of synchronous chaos. The pioneering work by Fujisaka and Yamada [1] demonstrates that chaos in coupled oscillators is synchronized when its maximal Lyapunov exponent is positive while the others are negative. However, the linear analysis is not sufficient to synchronous chaos since the higher-order perturbations are inevitably involved in chaos. Afraimovich et al. [2-4] show that synchronization in two slight different oscillators or subsystems can be achieved by the diffusive coupling, where the differences between two solutions are analytically estimated and can be controlled to decay. However, the estimation and control techniques are very difficult to be applied to a high-dimensional coupled system. Another related and interesting topic is how a coupled system of oscillators is synchronized to periodic states by chaotic driving signals. See Refs. [5,6] for details.

Synchronous chaos is one of most interesting topics on spatial extended systems. When many identical maps are coupled to a larger system (coupled map lattices or CML), the system can exhibit a variety of spatial configurations, such as kink-antikinks and wavelike patterns etc. [7]. Moreover, these configurations can also present local chaos on each map. In other words, a spatial regular configuration may admit temporal chaos. This phenomena seems to be contradictive to the initial sensitivity of chaos but it does exist. For example, the chaotic surface waves in Faraday experiments [8]. Let us begin with the simplest case: whether or not a spatial uniform configuration inherit the dynamics of the local map? For periodic motions, the answer is true. The periodic synchronization does exist. For chaotic motion, the synchronization can also be observed, which is more or less out of general intuition.

We will study synchronous chaos in two coupling Logistic maps in this paper. The approach developed here is not restricted to a two-dimensional case and could be extended to higher-dimensional CMLs. Our working model is as follows:

\footnotetext{
${ }^{*}$ Corresponding author.

E-mail address: hgw@Inm.imech.ac.cn (G.-w. He).
} 


$$
\left\{\begin{array}{l}
x_{t+1}=(1-\epsilon) f\left(x_{t}\right)+\epsilon f\left(y_{t}\right) \\
y_{t+1}=(1-\epsilon) f\left(y_{t}\right)+\epsilon f\left(x_{t}\right)
\end{array}\right.
$$

where $\epsilon$ is a coupling parameter with $0 \leqslant \epsilon \leqslant 1, f$ denotes a local map, which is taken as the Logistic map: $f(x)=1-a x^{2},(0 \leqslant a \leqslant 2)$. In Section 2 , the existence and stability of the synchronous chaos are discussed. In Section 3 , the structural stability on synchronous chaos is addressed. With those results, it is concluded that the synchronous chaos can be observed. In Section 4, it is shown that the synchronous chaos is a pseudo-orbit of the local map so that its dynamics can be described by the local map. The final section is devoted to the conclusions and discussions.

\section{Existence and stability of synchronous chaos}

It can be verified directly that the synchronized motion $x_{t}=y_{t}=z_{t}$, where $z_{t+1}=f\left(z_{t}\right)$, is an uniform solution of (1), no matter whether the motion $z_{t}$ is periodic or chaotic. This is because of the diffusive coupling in (1). The central problem is the stabilities of synchronous chaos. Due to its initial sensitivity, the orbits of the uniformly chaotic motion are never stable in phase space. However, the uniform configuration may be insensitive to its initial configuration so that the configuration is stable. In other words, the derivation from the synchronous configuration can be controlled, although the orbit $z_{t}$ itself is chaotic. Noting that the uniform motion $\left(z_{t}, z_{t}\right)$ is always located on the diagonal line of the phase space $\left(x_{t}, y_{t}\right)$, the transverse derivation to the diagonal line indicates the stability of the synchronous chaos.

Suppose that $\left(x_{t}, y_{t}\right)$ is a perturbed solution of (1) to synchronization $\left(z_{t}, z_{t}\right)$ by $\left(\delta x_{t}, \delta y_{t}\right)$, that is, $\delta x_{t}=x_{t}-z_{t}$, $\delta y_{t}=y_{t}-z_{t}$. Then

$$
\left(\begin{array}{l}
\delta x_{t+1} \\
\delta y_{t+1}
\end{array}\right)=E\left(\begin{array}{l}
f\left(x_{t}\right) \\
f\left(y_{t}\right)
\end{array}\right)-\left(\begin{array}{l}
f\left(z_{t}\right) \\
f\left(z_{t}\right)
\end{array}\right)=E\left(\begin{array}{c}
f\left(x_{t}\right)-f\left(z_{t}\right) \\
f\left(y_{t}\right)-f\left(z_{t}\right)
\end{array}\right)
$$

where $E$ is a diffusive matrix of the coefficients in (1):

$$
E=\left(\begin{array}{cc}
1-\epsilon & \epsilon \\
\epsilon & 1-\epsilon
\end{array}\right)
$$

Its eigenvalues and unitary eigenvectors are $\lambda_{1}=1$ and $\lambda_{2}=1-2 \epsilon$ separately and

$$
v_{1}=\frac{1}{\sqrt{2}}\left(\begin{array}{l}
1 \\
1
\end{array}\right), \quad v_{2}=\frac{1}{\sqrt{2}}\left(\begin{array}{c}
1 \\
-1
\end{array}\right) \text {. }
$$

The Taylor's expansion of the local map $f$ leads to

$$
\left(\begin{array}{l}
\delta x_{t+1} \\
\delta y_{t+1}
\end{array}\right)=E\left(\begin{array}{l}
f^{\prime}\left(z_{t}\right) \delta x_{t}+\frac{1}{2} f^{\prime \prime}\left(z_{t}\right) \delta x_{t}^{2} \\
f^{\prime}\left(z_{t}\right) \delta y_{t}+\frac{1}{2} f^{\prime \prime}\left(z_{t}\right) \delta y_{t}^{2}
\end{array}\right)=f^{\prime}\left(z_{t}\right) E\left(\begin{array}{c}
\delta x_{t} \\
\delta y_{t}
\end{array}\right)+\frac{1}{2} f^{\prime \prime}\left(z_{t}\right) E\left(\begin{array}{c}
\delta x_{t}^{2} \\
\delta y_{t}^{2}
\end{array}\right)
$$

where $f^{\prime}\left(z_{t}\right)=-2 a z_{t}, f^{\prime \prime}\left(z_{t}\right)=-2 a$.

The first order perturbation can be calculated in terms of the eigenvectors of $E$ :

$$
\left(\begin{array}{l}
\delta x_{t} \\
\delta y_{t}
\end{array}\right)=b_{t} v_{1}+c_{t} v_{2}=\frac{1}{\sqrt{2}}\left(\begin{array}{l}
b_{t}+c_{t} \\
b_{t}-c_{t}
\end{array}\right),
$$

where the coefficient $b_{t}$ is the magnitude of the projection of the perturbation $\left(\delta x_{t}, \delta y_{t}\right)$ to the diagonal eigenvector $v_{1}$, and $c_{t}$ is the magnitude of the projection of $\left(\delta x_{t}, \delta y_{t}\right)$ to the transverse eigenvector $v_{2}$, which indicates the transverse deviation. That is

$$
\frac{1}{\sqrt{2}} b_{t}=\frac{x_{t}+y_{t}}{2}-z_{t}, \quad \frac{1}{\sqrt{2}} c_{t}=\frac{1}{2}\left(x_{t}-y_{t}\right) \text {. }
$$

Similarly, the second order perturbation can be calculated as

$$
\left(\begin{array}{l}
\delta x_{t}^{2} \\
\delta y_{t}^{2}
\end{array}\right)=\frac{1}{\sqrt{2}}\left(b_{t}^{2}+c_{t}^{2}\right) v_{1}+\sqrt{2} b_{t} c_{t} v_{2} .
$$


Then, the perturbation at the next time step can be formulated as follows:

$$
\begin{aligned}
\left(\begin{array}{l}
\delta x_{t+1} \\
\delta y_{t+1}
\end{array}\right) & =f^{\prime}\left(z_{t}\right) E\left(b_{t} v_{1}+c_{t} v_{2}\right)+\frac{1}{2} f^{\prime \prime}\left(z_{t}\right) E\left(\frac{1}{\sqrt{2}}\left(b_{t}^{2}+c_{t}^{2}\right) v_{1}+\sqrt{2} b_{t} c_{t} v_{2}\right) \\
& =\left[f^{\prime}\left(z_{t}\right) b_{t} \lambda_{1}+\frac{1}{2} f^{\prime \prime}\left(z_{t}\right) \frac{1}{\sqrt{2}}\left(b_{t}^{2}+c_{t}^{2}\right)\right] v_{1}+\left[f^{\prime}\left(z_{t}\right)+\frac{1}{\sqrt{2}} f^{\prime \prime}\left(z_{t}\right) b_{t}\right] c_{t} \lambda_{2} v_{2},
\end{aligned}
$$

that is,

$$
\left\{\begin{array}{l}
b_{t+1}=f^{\prime}\left(z_{t}\right) b_{t}+\frac{1}{2 \sqrt{2}} f^{\prime \prime}\left(z_{t}\right)\left(b_{t}^{2}+c_{t}^{2}\right) \\
c_{t+1}=\left[f^{\prime}\left(z_{t}\right)+\frac{1}{\sqrt{2}} f^{\prime \prime}\left(z_{t}\right) b_{t}\right] \lambda_{2} c_{t} .
\end{array}\right.
$$

Eq. (2) expresses the perturbations of (1) exactly in terms of the eigenvectors of the linear matrix in (1). Noting that the second equation is linear with respect to $c_{t}$, an upper bound on $c_{t}$ can be formulated immediately.

Noting that $\left|b_{t}\right| \leqslant 2,\left|c_{t}\right| \leqslant 2,\left|f^{\prime}\left(z_{t}\right)\right| \leqslant 2 a,\left|f^{\prime \prime}\left(z_{t}\right)\right| \leqslant 2 a$, we obtain

$$
\left|c_{t+1}\right| \leqslant 4 a|1-2 \epsilon|\left|c_{t}\right| \text {. }
$$

Therefore, if $4 a|1-2 \epsilon|<1, c_{t}$ will decay to zero as $t$ goes to infinite. That is, the synchronous chaos is stable.

An alternative method of estimating $c_{t}$ is to calculate the Lyapunov exponents of (2) [9]:

$$
\begin{aligned}
& \rho_{1}=\lim _{N \rightarrow \infty} \frac{1}{N} \ln \left(\prod_{t=1}^{N}\left|f^{\prime}\left(z_{t}\right)\right|\right), \\
& \rho_{2}=\lim _{N \rightarrow \infty} \frac{1}{N} \ln \left(\prod_{t=1}^{N} \lambda_{2}\left|f^{\prime}\left(z_{t}\right)+\frac{1}{\sqrt{2}} f^{\prime \prime}\left(z_{t}\right) b_{t}\right|\right) .
\end{aligned}
$$

Here $\rho_{1}$ is the maximum Lyapunov exponent of (1) and also the Lyapunov exponent of the local map. $\rho_{1}<0$ is corresponding to periodic motions, and $\rho_{1}>0$ to chaotic ones. $\rho_{2}$ is the transversal Lyapunov exponent, which indicates the expanding rate of the transverse derivation perpendicular to the synchronous direction. When $\rho_{2}<0$, the transverse derivation $c_{t}$ will decrease to zero and thus the synchronous motions are stable. Therefore, $\rho_{1}>0$ and $\rho_{2}<0$ imply a stable synchronous chaos. Since $c_{t+1}$ is linearly dependent on $c_{t}$ in this case, the above analysis is exact.

In our model (1), $\epsilon$ can be chosen so close to 0.5 that $\lambda_{2}$ is enough small. In this case, $\rho_{2}<0$. Therefore, synchronized chaos can be realized in Eq. (1).

\section{Structure stability of synchronous chaos}

If there are some slight differences among local maps, can synchronous chaos be still observed? or does synchronous chaos exist for the coupled system of the slight different local maps? This problem is involved in structure stability of synchronization. In fact, it is impossible to have identical local maps in practice so that we have to deal with parameter perturbations.

We consider the coupled system of two slight different Logistic maps:

$$
\left\{\begin{array}{l}
x_{t+1}=(1-\epsilon) f\left(x_{t}, a_{1}\right)+\epsilon f\left(y_{t}, a_{2}\right) \\
y_{t+1}=(1-\epsilon) f\left(y_{t}, a_{2}\right)+\epsilon f\left(x_{t}, a_{1}\right)
\end{array}\right.
$$

where $f\left(x, a_{i}\right)=1-a_{i} x^{2}, 0 \leqslant a_{i} \leqslant 2$ and $\left|a-a_{i}\right| \leqslant s / 2(i=1,2), s$ is a small positive number.

We examine the difference between $x_{t}$ and $y_{t}$ :

$$
\begin{aligned}
x_{t+1}-y_{t+1} & =(1-2 \epsilon)\left[f\left(x_{t}, a_{1}\right)-f\left(y_{t}, a_{2}\right)\right]=(1-2 \epsilon)\left[f\left(x_{t}, a_{1}\right)-f\left(x_{t}, a_{2}\right)+f\left(x_{t}, a_{2}\right)-f\left(y_{t}, a_{2}\right)\right] \\
& =(1-2 \epsilon)\left[f_{a}^{\prime}\left(x_{t}, a_{3}(t)\right)\left(a_{1}-a_{2}\right)+f_{x}^{\prime}\left(\xi_{t}, a_{2}\right)\left(x_{t}-y_{t}\right)\right],
\end{aligned}
$$

where the mean value theorem is employed, and

$$
\begin{array}{ll}
f_{a}^{\prime}\left(x_{t}, a_{3}(t)\right)=-x_{t}^{2} & \left(a_{1} \leqslant a_{3}(t) \leqslant a_{2}\right), \\
f_{x}^{\prime}\left(\xi_{t}, a_{2}\right)=-2 a_{2} \xi_{t} & \left(x_{t} \leqslant \xi_{t} \leqslant y_{t}\right) .
\end{array}
$$


Noting that $f$ is the Logistic map, we have

$$
\left|f_{a}^{\prime}\left(x_{t}, a_{3}(t)\right)\right| \leqslant 1, \quad\left|f_{x}^{\prime}\left(\xi_{t}, a_{2}(t)\right)\right| \leqslant M \leqslant 4,
$$

where $M$ is a constant. Therefore,

$$
\begin{aligned}
& \left|x_{t+1}-y_{t+1}\right| \leqslant|1-2 \epsilon|\left[\left|f_{a}^{\prime}\left(x_{t}, a_{3}(t)\right)\left(a_{1}-a_{2}\right)\right|+\left|f_{x}^{\prime}\left(\xi_{t}, a_{2}(t)\right)\left(x_{t}-y_{t}\right)\right|\right] \\
& \quad \leqslant|1-2 \epsilon|\left[s+M\left|x_{t}-y_{t}\right|\right] \leqslant s|1-2 \epsilon|\left|\frac{1-[M(1-2 \epsilon)]^{t}}{1-M(1-2 \epsilon)}\right|+|M(1-2 \epsilon)|^{t+1}\left|x_{0}-y_{0}\right| .
\end{aligned}
$$

If $|M(1-2 \epsilon)|<1$, we obtain the following estimation at very large $t$ :

$$
\left|x_{t+1}-y_{t+1}\right| \leqslant \frac{|1-2 \epsilon| s}{1-M(1-2 \epsilon)} \text {. }
$$

Thus, if the difference between two nonlinear parameters is small enough, the difference between $x_{t}$ and $y_{t}$ still remains small. This result is hold only for the coupling parameter region: $|M(1-2 \epsilon)|<1$ and for all initial conditions. In fact, it implies nearly synchronous chaos but not an exact synchronous one. This shows that the slight perturbations of parameters in local maps do not change synchronization. That is, synchronization is structurally stable.

\section{Shadows of synchronous chaos}

The dynamics of the exactly synchronous chaos, where $x_{t}=y_{t}$, can be described by its local maps. The next question is how to describe the dynamics of the nearly synchronous chaos, where $x_{t}$ and $y_{t}$ are slight different. In this section, we will show that the mean of the nearly synchronous chaos can be tracked by the local maps.

It is known that $c_{t}$ is the distance from the point $\left(x_{t}, y_{t}\right)$ to the diagonal line $y=x$ in the phase plane. Thus, the difference between $x_{t}$ and $y_{t}$ is just the transverse derivation of the nearly synchronous chaos from the exact synchronous orbits. Noting that the interaction of the transverse deviation with the diagonal line is the point $\left(\frac{x_{t}+y_{t}}{2}, \frac{x_{t}+y_{t}}{2}\right)$, we can use the spatial mean value of (1) to describe the dynamics of the synchronized chaos.

We will show that the mean $\left(x_{t}+y_{t}\right) / 2$ is a $\epsilon$-pseudo-orbit of the local map $f$ if and only if $\left(x_{t}, y_{t}\right)$ is the $\delta$-synchronization, where $\epsilon=a \delta^{2} / 4$. This can be achieved by calculating

$$
\left|\frac{x_{t+1}+y_{t+1}}{2}-f\left(\frac{x_{t}+y_{t}}{2}\right)\right|=\left|\frac{f\left(x_{t}\right)+f\left(y_{t}\right)}{2}-f\left(\frac{x_{t}+y_{t}}{2}\right)\right|=\frac{1}{4} a\left|x_{t}-y_{t}\right|^{2} \text {. }
$$

From the above equations, it is easy to see that

$$
\left|\frac{x_{t+1}+y_{t+1}}{2}-f\left(\frac{x_{t}+y_{t}}{2}\right)\right| \leqslant \epsilon
$$

holds if and only if $\left|x_{t}-y_{t}\right| \leqslant \delta$.

It is reasonable to assume that chaotic attractors of the local maps are compact hyperbolic invariant sets. By Shadow lemma [9], there exists a real orbit $\left\{w_{t+1}=f\left(w_{t}\right)\right\}$ of the local map such that $\left(x_{t}+y_{t}\right) / 2$ can be shadowed by $\left\{w_{t}\right\}$, that is

$$
\left|\frac{x_{t}+y_{t}}{2}-w_{t}\right| \leqslant s
$$

where $s$ is a small positive number. Therefore, when $\left(x_{t}, y_{t}\right)$ is nearly synchronized, its dynamics can be described approximately by the corresponding local map; When $\left(x_{t}, y_{t}\right)$ exactly synchronized with $\delta=0$, it is just a real orbit of the local map. The pseudo-orbits $\left(x_{t}+y_{t}\right) / 2$ can be served as the descriptions of synchronized motions.

\section{Discussions and conclusions}

The stability of the synchronous configurations is investigated in this paper. When synchronous motions are periodic, their stabilities can be determined by the Lyapunov exponents; When synchronous motions are chaotic, linear analysis is not sufficient and nonlinear perturbations have to be considered. The method developed in this paper is to estimate the transverse derivations normal to the synchronous configurations. The expanding rates of the transverse derivations can be determined by the transverse Lyapunov exponents. The negative transverse Lyapunov exponents imply stabilities of synchronous configuration with temporal chaos. 
The diffusive coupling admits all synchronous motions. Due to the diffusive coupling, the eigenvector for the maximal Lyapunov exponent is corresponding to the configuration of synchronized motions. In this case, it possibly happens that all other Lyapunov exponents are negative while the maximum one is positive. Thus, synchronous chaos can be observed. However, if the synchronous configuration does not coincide with the eigenvector for the maximum Lyapunov exponent, synchronous chaos may leads to that some other Lyapunov exponents are positive. In this case, other spatial modes can be excited so that the synchronization disappears. For example, the convective coupling does not show the synchronous chaos [10]. More generally, the regular spatial configurations with temporal chaos can be observed only if the eigenvectors for the positive Lyapunov exponent are corresponding to the configurations of synchronized motions and the transverse derivations normal to the configurations have to be decaying. For example, a single wave in CML does not coincide with the eigenvector for the positive Lyapunov exponent so that it never exhibits chaos [11].

Synchronization is structurally stable. When the slight different local maps on units are coupled, a nearly synchronous motion can be still observed. This is because small perturbations to local maps do not block decrease of the transverse derivation. In other words, the transverse Lyapunov exponents are insensitive to local maps. A similar discussion can also be carried out on the coupling parameters.

The dynamics of synchronous chaos can be described by its local map. We have shown that $\left(x_{t}+y_{t}\right) / 2$ is a pseudoorbit of the local map. Therefore, for the hyperbolic maps, it can be shadowed by the real orbit of the local map. On the other hand, synchronization can be determined by whether or not $\left(x_{t}+y_{t}\right) / 2$ is a pseudo-orbit of the local map.

Our working model is two-dimensional. However, the methods suggested in the present paper are not restricted to low-dimensional cases. They can be used for the higher-dimensional coupled map lattices. It has been found by our numerical simulation that higher-dimensional CMLs do exhibit nearly synchronous chaos. From synchronous chaos, nonuniform configuration will be developed via bifurcation from the wavelike patterns, which are either the longest or shortest spatial wavelength $[12,13]$. The stabilities of the regular spatial configurations with temporal chaos can also be investigated by the transverse derivations.

\section{Acknowledgements}

G.-w. He acknowledges the support of the National Committee of Science and Technology, PR China under the program "Nonlinear Science".

\section{References}

[1] Fujisaka H, Yamada T. Stability theory of synchronized motion in coupled-oscillators systems. Prog Theor Pyhs 1983;69(1):3247.

[2] Affraimovich VS, Verichev NN, Rabinovich MI. Stochastic synchronization of oscillation in dissipation systems. Izvestiya Vysshikh Uchebnykh Zavedenij, Radiofizika 1986;29(9):1050-60, in Russian.

[3] Rodrigues HM. Uniform ultimate boundsness and synchronization. In: Technical Report CDSN94-160. Center for Dynamical System and Nonlinear Studies, Georgia Tech., 1994.

[4] Affraimovich VS, Rodrigues HM. Uniform ultimate boundsness and synchronization for nonautonomous equations, In: Technical Report CDSN 94-202. Center for Dynamical System and Nonlinear Studies, Georgia Tech., 1994.

[5] Pecora LM, Carroll TL. Synchronization in chaotic systems. Phys Rev Lett 1990;64(8):821-4.

[6] Pecora LM, Carroll TL. Driving systems with chaotic signals. Phys Rev A 1991;44(4):2374-83.

[7] Crutchfield JP, Kaneko K. Phenomenology of spatio-temporal chaos. In: Directions in Chaos. Singapore: World Scientific; 1987. p. 272-353.

[8] Miles J, Henderson D. Parametrically forced surface waves. Annu Rev Fluid Mech 1990;22:143-65.

[9] Guckenheimer J, Holmes P. Nonlinear oscillation, dynamical systems, and bifurcations of vector fields. New York: Springer; 1983. p. $250-251$.

[10] He G, Cao L, Li J. Convective coupled maps for simulating spatiotemporal chaos in flows. Acta Mech Sin 1995;11(1):1-7.

[11] He G, Lambert A, Lima R. Wavelike patterns in one-dimensional coupled map lattices. Physica D 1997;103:404-11.

[12] Lambert A, Lima R. Stability of wavelengths and spatio-temporal intermittency. Physica D 1994;71:390-411.

[13] Heagy JF, Pecora LM, Carroll TL. Short wavelength bifurcations and size instabilities in coupled oscillator systems. Phys Rev Lett 1995;74(21):4185-8. 